\title{
Urgences
}

\section{Le manuscrit comme lieu de dialogue : Alfred DesRochers}

\section{Richard Giguère}

Numéro 24, juillet 1989

Le manuscrit sous l'angle

URI : https://id.erudit.org/iderudit/025529ar

DOI : https://doi.org/10.7202/025529ar

Aller au sommaire du numéro

Éditeur(s)

Urgences

ISSN

0226-9554 (imprimé)

1927-3924 (numérique)

Découvrir la revue

Citer cet article

Giguère, R. (1989). Le manuscrit comme lieu de dialogue : Alfred DesRochers.

Urgences, (24), 25-34. https://doi.org/10.7202/025529ar d'utilisation que vous pouvez consulter en ligne.

https://apropos.erudit.org/fr/usagers/politique-dutilisation/ 


\section{Le manuscrit comme lieu de dialogue: Alfred DesRochers}

\section{Richard Giguère, Université de Sherbrooke}

En acceptant l'invitation d'André Carpentier et de René Lapierre à participer au colloque "Le manuscrit sous l'angle de la génétique littéraire", je me suis fixé un double objectif. D'abord celui de présenter ce qui, à mon avis, constitue la caractéristique la plus intéressante et la plus originale des manuscrits de deux recueils de poèmes d'Alfred DesRochers, L'offrande aux vierges folles (1928) et $\dot{A}$ l'ombre de l'Orford (1920), c'est-àdire le dialogue animé et instructif qui prend place sur les manuscrits eux-mêmes entre Émile Coderre et DesRochers d'une part, et entre DesRochers et Louis Dantin d'autre part. Mon deuxième objectif est d'aider à remettre en circulation des auteurs et des textes des décennies vingt et trente qui ont été plus ou moins oubliés ces dernières années au Québec. La publication des actes de ce colloque, ajoutée à la publication des éditions critiques d'Un homme et son péché de ClaudeHenri Grignon, des Demi-civilisés de Jean-Charles Harvey et des deux premiers recueils de DesRochers, contribuera sans doute à sortir ces auteurs du purgatoire où ils ont été relégués.

\section{Les manuscrits de L'offrande aux vierges folles (1919-1928)}

Il me faut d'abord dire quelques mots des manuscrits de L'offrande aux vierges folles et d'À l'ombre de l'Orford retrouvés dans le fonds DesRochers déposé aux Archives nationales du Québec, centre régional de l'Estrie, à Sherbrooke. Je ne tiens pas compte ici des nombreuses ébauches de poèmes trouvées dans les papiers de DesRochers, mais uniquement de manuscrits substantiels et constitués en ensemble.

Au sujet de L'offrande aux vierges folles, d'abord publié en 1928, nous avons mis la main sur trois manuscrits importants représentant différentes étapes de l'écriture et de la réécriture du recueil sur une période de dix ans, de 1919 à 1928. Le premier manuscrit est un cahier ligné (vingt-cinq lignes rouges à la page) de $18 \times 25 \mathrm{~cm}$, contenant cinquante-quatre pages (treize feuilles de $36 \mathrm{~cm}$ pliées en deux et agrafées, plus un feuillet de "Table des poèmes", le tout de type "petit cahier d'écolier"). Ce manuscrit est écrit de la main de DesRochers, 
avec une plume à l'encre (quatre teintes différentes de bleu et de noir), et contient des corrections et des notes rédigées aussi par DesRochers, mais à la mine de plomb. Les deux premières pages du manuscrit comprennent une "Table des poèmes" énumérant les titres et les numéros correspondants de quarante-quatre poèmes, datés du 29 septembre 1919 - DesRochers avait dix-sept ans! - au 22 juin 1924.

Dix-huit de ces poèmes ont été écrits par DesRochers alors qu'il fréquentait le Collège séraphique de Trois-Rivières (de septembre 1918 à juin 1921), vingt-six alors qu'il travaillait à différents emplois dans la région de Sherbrooke avant de se marier, le 20 mai 1925, et d'entrer à plein temps au journal La tribune en juillet de la même année. Dans les marges de gauche ou de droite des poèmes, DesRochers a écrit à la mine de plomb les commentaires suivants (qui datent sans doute de l'automne 1928:
- À refaire
(13 poèmes)
- Nul
(13 poèmes)
- OK
(10 poèmes)
- «Filler"
- À peu près nul
(2 poèmes)
- OK avec corrections
(1 poème)
(1 poème)
- À corriger
(1 poème)

Les poèmes retenus par DesRochers dans L'offrande aux vierges folles - près d'une quinzaine sur les quarante-quatre du manuscrit - sont accompagnés de sections qui, sauf exception, n'ont pas été retenus dans le recueil de 1928: "Paysages" - trois poèmes, "Visions rustiques" - un poème, "Visions sylvestres" - un poème, "Visions tristes - un poème, "Visions d'adolescents" - trois poèmes, "Visions" - un poème, "Visions historiques" - deux poèmes, "Agavé" - un poème.

Le deuxième manuscrit est un cahier de $18 \times 23,5 \mathrm{~cm}$ (vingt-six lignes bleues à la page), également de type "petit cahier d'écolier". II comprend vingt-huit pages (six feuilles de 36 $\mathrm{cm}$ pliées en deux et agrafées - vingt-quatre pages, plus deux feuilles coupées - quatre pages) et contient vingt-deux poèmes et une suite poétique. Les poèmes de ce deuxième cahier, au contraire de ceux du premier, ne sont pas numérotés ni datés, et ils ne sont pas précédés d'une table des matières. (Nous savons, par recoupements avec les autres manuscrits, que ces poèmes ont été écrits en 1925 ou en 1926.) Un peu plus du tiers 
des textes de ce manuscrit et la suite poétique intitulée "Petits airs sur deux notes, pour Elles toutes", tous écrits à la plume (encre noire uniforme), sont repris dans L'offrande, mais avec de nombreuses variantes. La plupart des titres ont été conservés, mais non pas les titres des sections, sauf une exception: "Religiosité - deux poèmes, "Le jardin secret" - trois poèmes, "Paysages" - un poème, "Sonnets bourgeois" - deux poèmes, "Exotisme sur chromos" - deux poèmes, "Verbalisme" - trois poèmes.

Parmi les poèmes retenus dans L'offrande aux vierges folles, le plus célèbre porte le titre "Atavisme". Il est suivi, entre parenthèses, du titre de section "Paysages". DesRochers a rayé le sommet d'un grand trait en zigzag et a écrit dans la marge de droite: “(À refaire)". Ce sonnet est en fait la première version connue du célèbre liminaire du "Cycle des bois et des champs", intitulé plus tard "Je suis un fils déchu" (quarante et un vers) et inséré dans $\dot{A}$ l'ombre de l'Orford. Six poèmes de ce cahier comprennent de nombreuses "Variantes" en bas de pages. Un poème - «Orientale. (Le jardin secret)" - porte la mention "Offrande aux vierges folles" dans la marge de droite, ce qui signifie que DesRochers a songé à ce titre pour son recueil peut-être dès 1926, ou même avant. En travers d'un long poème intitulé "Ferveur décadente", DesRochers a écrit à la mine de plomb, en gros caractères, "Mysticisme sentimental", suivi de la mention "OK"; le poème et le titre seront repris dans L'offrande. Enfin, le sonnet qui donne son titre au recueil, "L'offrande aux Vierges folles", est accompagné de la mention "à refaire" dans la marge de droite et est suivi, dans la moitié inférieure de la page, de pas moins de vingt-cinq variantes (des vers entiers) pour un texte de quatorze vers.

Le troisième manuscrit comprend neuf feuillets tapés à la machine par DesRochers (feuillets de $21 \times 27,5 \mathrm{~cm}$, tapés tantôt d'un seul côté, tantôt des deux côtés). II contient quatorze poèmes et une suite poétique, envoyés par DesRochers à son ami le poète Émile Coderre (avec une lettre de mars 1927) pour commentaires, corrections et suggestions. Des quinze titres, DesRochers a retenu douze poèmes et la suite poétique «Petits airs sur deux notes" dans L'offrande (neut des douze sonnets de la section "Sonnets", deux des quatre titres de la section "Pour elles toutes", un des cinq poèmes de la section "Paysages" et "Mysticisme sentimental" de la section "Désespérance romantique"). 
Des trois manuscrits de L'offrande aux vierges folles, ces poèmes tapés à la machine et envoyés à Émile Coderre en mars 1927 sont les plus instructifs car, dans la marge de gauche des neuf feuillets, perpendiculairement au texte, DesRochers écrit à la mine de plomb des notes s'adressant à son ami Coderre, lui pose quelques questions, lui demande son avis sur certains passages. Dans la marge de droite et dans les marges du haut et du bas des feuillets, Coderre écrit ses commentaires, fait des suggestions, répond aux questions de DesRochers ou lui pose des questions à son tour (les notes sont écrites à la mine de plomb la plupart du temps, quelquefois au crayon rouge). Le seul poème signé et daté par DesRochers est "Grandiloquence". Au bas du feuillet, en partant de la marge de gauche, DesRochers a tapé sur deux lignes et souligné: “7 et 8 mars 1927 / Sherbrooke". Si on ajoute à ces deux lignes le mot "Finale", tapé par DesRochers au haut du feuillet et également souligné, on peut supposer avec passablement de certitude qu'il s'agit du dernier feuillet du manuscrit envoyé par DesRochers à Coderre en mars 1927. Le dialogue de Coderre et de DesRochers, dans les marges de ce manuscrit, annonce celui qu'auront deux ans plus tard Dantin et DesRochers au sujet des poèmes d'À l'ombre de l'Orford. Les commentaires de Coderre, plus généraux, portent moins sur des points de détail et de technique du vers que ceux de l'échange entre Dantin et DesRochers. L'autre différence majeure, c'est que DesRochers, lors de la publication de L'offrande, en novembre 1928, a peu tenu compte des corrections et suggestions de Coderre alors qu'il agira tout autrement avec les notes de Dantin portant sur les poèmes de l'Orford.

\section{Le dlalogue Dantin-DesRochers}

C'est le 20 janvier 1929, deux mois après la publication de L'offrande aux vierges folles, que DesRochers demanda à Louis Dantin la permission de lui soumettre les poèmes de son futur recueil afin d'avoir son opinion et ses commentaires:

Je sais que votre temps est hypothéqué et que vous avez bien autre chose à faire que de donner des cours de littérature par correspondance (entre parenthèses, si j'étais vous, je le ferais; je sais bon nombre d'écrivains canadiens qui seraient prêts à payer pour obtenir votre avis). Toutefois je vous soumets deux échantillons de mon projet. S'il vous semble valoir quelque chose, vous n'aurez pas besoin de me le dire. "Qui ne dit mot consent». Si ça ne vaut rien, écrivez-moi simplement le mot 
«rotten " sur une carte et je saurai à quoi m'en tenir. Vous acceptez? (DesRochers à Dantin, 20/1/1929, ANQ-S)

À sa lettre, DesRochers joignait deux pièces: "La chanson de la fileuse" (devant faire partie du "Cycle des demeures et des routes") et "Hymne au Vent du Nord".

Le 3 février 1929, Dantin accusa réception des poèmes. Enthousiaste, il déclarait qu'il s'était donné le plaisir de les étudier en détail et de les annoter. Le 16 mars 1929, DesRochers soumit à Dantin de nouveaux poèmes, "Soir d'été à Saint-Denis de Brompton", "Prière au bọn Dieu des gens frustes de chez nous", "Ma province aux noms exotiques" et, le 5 avril, à la fin de sa lettre, le poète recopia de mémoire les sonnets "CityHôtel» et "Sur la Totrôde". Dantin retourna ces poèmes le 16 avril en mentionnant qu'il ne s'était pas permis cette fois de les couvrir de "griffonnages", "qui n'auraient eu d'ailleurs aucune raison d'être". II se contentait de les commenter et de critiquer plus particulièrement «Ma province aux noms exotiques", qui devait à l'origine donner son titre au recueil. II suggéra au poète de changer ce titre. Le 29 juin 1929, DesRochers fait parvenir à Dantin les quatorze sonnets de la Suite forestière. Dantin la renvoie le 22 juillet avec ses commentaires (sur le manuscrit, il n'a noté que "quelques vétilles", selon ses propres dires). Enfin, le 4 septembre 1929, alors que son recueil est déjà à l'imprimerie de La tribune, DesRochers soumet à Dantin les quatorze sonnets du Cycle des champs, la "Finale" de la Suite forestière et, sous pli séparé, l' "Hymne au Vent du Nord" retravaillé. Dantin les renverra, annotés, le 29 septembre suivant, et DesRochers tiendra compte de ses corrections et suggestions et retravaillera ses poèmes, "au grand dam de son imprimeur", dit-il.

DesRochers a donc envoyé à Dantin la presque totalité des poèmes d'À l'ombre de l'Orford (trente-trois poèmes dans l'édition de 1929). En fait, seuls le célèbre liminaire, "Je suis un fils déchu", et le sonnet d'ouverture dédié "À M. Alphonse Désilets" ne sont jamais mentionnés dans la correspondance. Nous n'avons malheureusement pas retrouvé tous ces poèmes avec les lettres. Certains ont pu être égarés au cours des ans, perdus dans les déménagements ou détruits par DesRochers. Notre manuscrit, composé uniquement de poèmes dactylographiés, est donc incomplet. Toutefois, malgré ces lacunes, le manuscrit d'À l'ombre de l'Orford est intéressant à plus d'un titre. D'abord, comme DesRochers incluait ses poèmes dans les 
lettres qu'il envoyait à Dantin, cela nous permet de dater approximativement la rédaction des textes. Mais en plus, les poèmes sont annotés par Dantin et souvent par le poète luimême, ce qui permet d'apprécier le rôle important que le critique Dantin joua auprès du jeune DesRochers. Par exemple, le manuscrit d' "Hymne au Vent du Nord" est couvert de notes et de commentaires de Dantin et, à lire le texte de base, on se rend compte que DesRochers a tenu compte en grande partie des suggestions du critique. Dans les marges du manuscrit des 14 sonnets du Cycle des champs, c'est un véritable dialogue qui prend forme entre le critique et le poète: on y retrouve d'une part les notes et commentaires de Dantin et d'autre part les corrections et les explications de DesRochers en réponse à ces commentaires. Pourtant, je le rappelle, le poète avait envoyé à Dantin ces quatorze sonnets alors que son recueil était déjà à l'imprimerie de La tribune. DesRochers prit quand même la peine de retravailler ses textes et, le 30 septembre 1929, il écrivit à Dantin:

Deux mots à l'épouvante - en pleine tourmente électorale pour vous dire que je vous suis très reconnaissant des corrections que vous m'avez suggérées à la Suite champêtre et que mon imprimeur est en amaudit" contre vous pour la même raison. J'ai repris presque tous les vers que vous condamniez. Avec quel succès? Je ne le sais plus. (DesRochers à Dantin, le 30/9/1929, ANQ-s)

D'autres extraits de lettres permettent de mesurer l'importance de Dantin comme mentor littéraire de DesRochers:

Mais vos annotations permettent de doubler la valeur de mes élucubrations. Vous verrez le a Vent du Nordn, quand je vous le renverrai corrigé. (DesRochers à Dantin, 11/4/1929, ANQ-S)

C'est moi qui suis votre débiteur. Voici, vous avez lu tout le manuscrit d'A l'ombre de l'Orford, vous avez fait des suggestions qui ont amélioré ces vers à 100 pour 100. (DesRochers à Dantin, 4/1/1930, ANQ-S)

L'intérêt premier de la lecture des variantes de l'édition critique des deux recueils de DesRochers, c'est ce dialogue vivant, rempli d'humour et de jeux de mots, qui s'engage entre DesRochers et Coderre au sujet de la moitié (quatorze sur trente) des poèmes de L'offrande d'une part, et particulièrement entre DesRochers et Dantin au sujet des deux tiers (vingt et un sur vingt-trois) des poèmes de l'Orford d'autre part. C'est du moins ce que j'ai tenté de conserver dans l'appareil des 
variantes en employant des formules telles que: "Dans la marge de droite, Dantin (ou Coderre) note que...", "remarque que...", "fait le commentaire suivant...", "demande à DesRochers si...", etc. et: "Sous ce commentaire, DesRochers écrit...", "répond...", "réplique...", etc. J'ai donc tenté, dans la mesure du possible, de préserver les caractéristiques de ce dialogue qui se déroule sur le manuscrit même et qui est, le temps et l'espace l'exigent, court et ad rem. Qu'il s'agisse de détails ou d'impressions d'ensemble, de petites fautes de grammaire ou de syntaxe (à peu près jamais d'orthographe) ou de contresens et d'erreurs majeures, de points techniques de versification ou de subtilités phonétiques, de discussions sur les anglicismes ou les canadianismes, tout est abordé dans ce dialogue. Les commentaires, les questions et réponses des interlocuteurs, les mots et les vers soulignés ou placés entre crochets ou entre parenthèses, quelquefois seulement un point d'interrogation ou d'exclamation, tout est là sur le manuscrit, inscrit de façon immédiate et vérifiable, pour peu qu'on se donne la peine de déchiffrer l'écriture et les codes de Coderre, Dantin et DesRochers.

À n'en pas douter, les avantages d'un dialogue sur le manuscrit même sont nombreux pour le travail d'interprétation des variantes, car ce dialogue nous fournit la plupart du temps les raisons des changements, modifications ou corrections apportés par DesRochers à son texte, suite aux notes, commentaires et suggestions de Coderre et Dantin. Je n'ai pas à me perdre en hypothèses et en suppositions pour expliquer les variantes, puisque DesRochers et ses deux lecteurs privilégiés discutent eux-mêmes de ces variantes, quelquefois dans les moindres détails. Les exemples les plus convaincants sont sans doute le long poème "Hymne au vent du nord" et la suite "À l'ombre de l'Orford".

\section{Compréhension de surface ou compréhension profonde}

On m'objectera peut-être qu'il s'agit d'une compréhension de surface du texte, que les variantes ne remettent pas en cause le sens profond et global des poèmes de DesRochers. Mais l'expérience me prouve au contraire que le dialogue DantinDesRochers en particulier, et aussi, dans une moindre mesure, le dialogue Coderre-DesRochers, amènent inévitablement celuici à exposer ses intentions, sa conception de la poésie, son "art poétique" en somme. DesRochers ne se contente pas 
d'accepter ou de réfuter les corrections ou suggestions de ses deux lecteurs. En manifestant son accord ou son désaccord face à certaines remarques, il est souvent obligé de s'expliquer en appuyant ses propos d'arguments de fond. (Et on sait par ailleurs, grâce à ses "Carnets" inédits et à une série d'articles et d'interviews publiés, que DesRochers est aussi un théoricien de la littérature qui a longuement réfléchi aux questions de versification, de poésie traditionnelle vs poésie moderne, de la fonction sociale du poète, des rapports entre la littérature et l'État, etc. $\left.{ }^{1}\right)$. Par le fait même, il dévoile ses intuitions et ses aspirations de poète dans le Québec des années vingt et trente, non seulement dans ses manuscrits, mais plus encore dans sa volumineuse correspondance avec Coderre et Dantin. C'est dans ces correspondances en particulier, mais aussi dans celles avec le critique Albert Pelletier, les poètes Rosaire DionLévesque, Alice Lemieux, Lucien Rainier, Simone Routier et Éva Sénécal, que se trouve l'exposé de la genèse des deux premiers recueils de DesRochers.

Toujours au sujet de la compréhension des textes, ce sont les différents manuscrits de L'offrande aux vierges folles et d'À l'ombre de l'Orford qui nous aident à saisir le principe d'organisation et la structure de ces recueils. Je prends comme exemple le manuscrit des quatorze sonnets de la suite "À l'ombre de l'Orford". Immédiatement sous le titre général de la suite, "À L'OMBRE DE L'ORFORD" écrit en majuscules, DesRochers a tapé à la machine, toujours en lettres majuscules, "AU TEMPS", suivi des quatorze sonnets qui sont placés l'un après l'autre sur les six feuillets du manuscrit, sonnets numérotés en chiffres romains et coiffés des titres suivants: "(AU TEMPS) / / - - / Des Clôtures / / -11- / De la Mise en Pacage / / -III- / Du Labour / /" etc. Cette suite est donc axée sur le temps, alors que l'autre suite du recueil, "La naissance de la chanson" (les sonnets portant sur les chantiers et la drave), est axée sur l'espace et le temps. C'est cette structure temporelle particulière, cet ordre séquentiel lié au travail des champs, non repris depuis 1929 dans les éditions successives d'À l'ombre de l'Orford, qui donne pourtant son sens véritable au titre

1 Voir mon article intitulé aAlfred DesRochers, théoricien et historien de la littératurem, dans A l'ombre de DesRochers. Le mouvement littéraire des Cantons de l'Est (1925-1950), Sherbrooke, La Tribune et les Éditions de l'Université de Sherbrooke, 1985, p. 149-174. 
général qui coiffe les deux suites de quatorze sonnets, "LE CYCLE DES BOIS ET DES CHAMPS" (cycle vient du grec kuklos qui veut dire "cercle"). Le cycle de la vie des paysans de l'Orford - mais non seulement de l'Orford - se répète chaque année, d'année en année: six mois dans les bois (les chantiers, du mois d'octobre au mois de mars), et six mois dans les champs (d'avril à septembre).

Permettez-moi un autre exemple, avant de conclure, portant sur la conception que se fait DesRochers du poète et de la poésie. À l'instar de plusieurs écrivains et intellectuels de sa génération, DesRochers est le premier de sa famille d'agriculteurs et d'hommes de chantiers à travailler comme journaliste, donc à ne pas exercer un travail manuel. D'où sa nostalgie, exprimée un peu partout dans sa poésie et dans ses articles, dans ses interviews et dans sa correspondance, pour ses ancêtres hommes de chantiers et coureurs des bois (son grandpère, son père et ses oncles). L'isotopie du travail manuel est chez lui omniprésente, même dans sa conception de l'écrivain et de l'écriture. Le poète mis en scène dans ses poèmes n'est pas un artiste mais un artisan, avec toute la dignité que DesRochers confère à ce mot (voir le "Dernier couplet" de la Suite forestière, CEuvres poétiques I, p. 92). Le poète "ouvre" des vers comme un artisan "ouvre" du bois. DesRochers parle même, dans une lettre adressée à Éva Sénécal, de «tordre quelques sonnets" pendant une soirée passée à la maison, comme un artisan "tord" un matériau, comme un sculpteur travaille la glaise pour en tirer une forme. D'où le plaisir de DesRochers, suite à une première rédaction de ses poèmes, à les travailler et à les retravailler. C'est-à-dire à les changer, à les modifier à la force des poignets, des muscles, c'est-à-dire à suer à la tâche, comme un ouvrier en plein travail ou comme un bûcheron dans les chantiers.

Ce que je retiens du contact privilégié que j'ai eu avec les manuscrits, les papiers personnels et la correspondance de DesRochers, c'est une leçon d'humilité. Voir de près et étudier ces manuscrits, comprendre, par la correspondance et les interviews menées avec les auteur-e-s de leur vivant, les (difficiles) conditions d'écriture de ces textes, cerner la problématique des variantes, vérifier la distance entre les intentions, les ambitions du jeune DesRochers et la réception de ses recueils par la critique de l'époque - voilà autant de phénomènes qui ont 
changé ma vision non seulement de la poésie de DesRochers, mais de toute la littérature québécoise de l'entre-deux-guerres, une période économiquement, socialement et culturellement déprimée. Les temps furent très durs pour les jeunes écrivains de cette époque, ceux que DesRochers se plaisait à appeler "la génération perdue»: Harry Bernard, Albert Pelletier, JeanCharles Harvey, Medjé Vézina, Éva Sénécal, Simone Routier qui fuira en Europe, Jovette Bernier qui passera à la radio, comme Claude-Henri Grignon et Robert Choquette. En tout, une dizaine de poètes, de romanciers et de critiques qui ne se remirent pas des durs coups de la Crise et furent éclipsés de la scène littéraire à la fin des années trente. On a l'impression, après la Deuxième Guerre mondiale, qu'une génération d'écrivains a été fauchée sur les champs de bataille tant elle est absente du paysage littéraire québécois, et que tout recommence. 